\title{
Sustained Voluntary Climbing Exercise Increases Erythrocyte Monocarboxylate Transporter 1 in Rats
}

\author{
Wataru AoI, Maiko Tsuzuki, Mitsuo FuJie, Soh IwASHITA, and \\ Masashige SUZUKI* \\ Laboratory of Exercise and Nutrition, Institute of Health and Sport Sciences, \\ University of Tsukuba, Tsukuba 305-8574, Japan
}

(Received September 22, 2001)

\begin{abstract}
Summary The effects of four weeks of climbing exercise on erythrocyte monocarboxylate transporter 1 (MCT1) content in rats were studied. The erythrocyte MCT1 content was significantly higher in the climbing group than in the sedentary group $(p<0.05)$. Blood lactate concentration was higher $(p<0.05)$, and the relationship between plasma and intra-erythrocyte lactate concentrations was positively correlated in the climbing group $(r=0.80, p<0.001)$. When erythrocytes of the climbing group were separated into young and old ones, young erythrocytes is a tendency to have more MCT1 than old erythrocytes (NS). The increase in erythrocyte MCT1 content by a sustained voluntary climbing exercise may contribute to the rapid lactate delivery to liver and oxidative tissues.
\end{abstract}

Key Words: MCT1, lactate, erythrocyte, climbing, rat

During exercise, lactate produced in contracting muscles is released into circulating blood and delivered to oxidative tissues, the liver and kidneys, in what is called the "extra-cellular lactate shuttle" [1]. In blood, lactate is vigorously transported across erythrocyte membranes $[2,3]$. Because lactate transport across the membranes depends on the plasma and intra-erythrocyte lactate gradient, elevation in plasma lactate concentration during exercise induces an increase in intra-erythrocyte lactate concentration $[2,3]$. The lactate transport between erythrocytes and plasma occurs predominantly via $\mathrm{H}^{+}$-monocarboxylate cotransporter 1 (MCT1) $[4,5]$, the isoform of which is found only in erythrocytes [6]. Therefore, we hypothesized that erythrocyte MCT1 content is up-regulated with increased membrane lactate transport. However, the effect of continuing exercise on erythrocyte MCT1 content has not been reported.

In the present study, MCT1 content in rat erythrocytes was determined after four

*To whom correspondence should be addressed. Tel.Fax.:+81-298-53-2733. E-mail: msuzuki@ taiiku.tsukuba.ac.jp 
weeks of a sustained climbing exercise that was developed in our laboratory for an animal model of voluntary resistance exercise $[7,8]$.

\section{MATERIALS AND METHODS}

Animals and experimental design. The experimental protocols for this study were approved by the Institutional Animal Care and Use Committee at the University of Tsukuba. Thirty male Sprague-Dawley rats aged 10 weeks were divided into sedentary and voluntary climbing exercise groups with 5 rats per each cage and cared for as previously described [7]. During the 4 weeks of the experimental period, food intake was equalized among the groups by meal-feeding at 7:30-8:30 and 19:30-20:30 with lights on from 6:00 to 18:00. The climbing group was allowed to voluntarily climb in a wiremesh tower cage $(20 \mathrm{~cm} \phi, 2 \mathrm{~m})$ as previously described [7, 8]. In the climbing group, the rats were video-monitored for $24 \mathrm{~h}$ /day every 2 weeks. The daily number of climbing and time of one round climbing were obtained from the monitoring records.

Measurement of lactate concentration. After 4 weeks, blood was collected from the tail vein at 21:00. Blood and plasma lactate concentrations were measured by a lactate analyzer (YSI-sports 1500, Yellow Spring Inst., OH), and erythrocyte lactate concentration was calculated with the following equation [9]:

$$
[\mathrm{La}]_{\mathrm{E}}=\left([\mathrm{La}]_{\mathrm{B}}-\left([\mathrm{La}]_{\mathrm{P}}(1-\mathrm{Hct})\right)\right) \mathrm{Hct}^{-1}
$$

where $[\mathrm{La}]_{\mathrm{E}}$ is the lactate concentration in the erythrocyte; $[\mathrm{La}]_{\mathrm{B}}$ is the measured wholeblood lactate concentration; $[\mathrm{La}]_{\mathrm{P}}$ is the measured plasma lactate concentration; Hct is the measured hematocrit value.

Preparation of protein sample. At 4 weeks after beginning of the experiment, blood samples were collected from abdominal main artery and rats were sacrificed under ether anesthesia. A portion of the blood was separated by Percoll solution (Pharmacia, Uppsala, Sweden) adjusted to the density of $1.100,1.105$, and $1.110 \mathrm{~g} / \mathrm{ml}$. Low density part was used as young erythrocyte and high density part was used as old erythrocyte. Erythrocyte ghosts were prepared as previously described [10]. After homogenization of the ghosts, the suspension was centrifuged at $14,000 \times g$ for $10 \mathrm{~min}$ at $4^{\circ} \mathrm{C}$, and the supernatant fluid was stored at $-50^{\circ} \mathrm{C}$ and used for immunoblot detection of MCT1.

Western blotting of MCT1. A polyclonal anti-MCT1 antibody was produced by a method similar to that of Carpenter et al. [11]. Using the antibody, erythrocyte MCT1 was assayed by a modified Western blotting analysis method in our laboratory as described in a previous report [12]. The solubilized erythrocyte membrane proteins were mixed with lysate buffer and sample buffer containing $0.05 \mathrm{M}$ Tris- $\mathrm{HCl}(\mathrm{pH} 6.8), 10 \%$ glycerol, $2 \%$ SDS, $5 \% 2 \beta$-mercaptoethanol and $0.05 \%$ bromophenol blue. The solubilized proteins in the buffers were boiled for $4 \mathrm{~min}$, then separated on $10 \%$ SDS-PAGE (Tefuko, Tokyo, Japan) directly. The separated proteins were electrophoretically transferred to nitrocellulose membrane (Millipore, Tokyo, Japan) using a transfer buffer containing $25 \mathrm{mmol} /$ liter Tris-base, $192 \mathrm{mmol} /$ liter glycine, $20 \%$ methanol and $0.02 \%$ SDS. The membranes were incubated in blocking buffer (3\% BSA in wash buffer containing 20 $\mathrm{mmol} /$ liter Tris-base, $150 \mathrm{mmol} /$ liter $\mathrm{NaCl} 0.01 \%$ Tween 20 ) at room temperature for 60 
min, then incubated overnight at $4{ }^{\circ} \mathrm{C}$ with the polyclonal anti-MCT1 antibody. After overnight incubation, the membranes were well washed in buffer, then incubated in $1 \%$ $\left[\mathrm{I}^{125}\right]$ protein A (IM144, Amersham, London, England) in blocking buffer at room temperature for $60 \mathrm{~min}$. The membranes were washed at room temperature for $60 \mathrm{~min}$ with agitation, changing the wash buffer every $15 \mathrm{~min}$. They were dried and exposed isotopic activity to an imaging-plate (BAS-SR 2025, Fuji-film, Tokyo, Japan) in a cassette (BASCassette 2025, Fuji-film). Band intensities were quantified by a laser image analyzer (BAS 5000, Fuji-film). The quantitated MCT1 content was corrected by the erythrocyte counts used for the analysis.

Statistical analysis. Values are expressed as the mean \pm SE. Two-way ANOVA was used to compare the erythrocyte MCT1 content for the age of erythrocytes and exercise. When ANOVA showed significant differences, Fisher's PLSD test was used. Linear regression analysis was used to calculate the correlation between plasma and intra-erythrocyte lactate concentrations. Other data were assessed with Student's $t$-test. Significant levels were set at $p<0.05$.

\section{RESULTS}

The averaged number of climbing 1 and 3 weeks after starting the experiment were 14.4 and 18.7 times/day. The respective time periods for one round climbing were 2.1 and $2.7 \mathrm{~min} /$ round. Table 1 shows the body weights and hematological parameters of the sedentary and climbing groups at 4 weeks. Body weight was significantly lower in the climbing group than in the sedentary group $(p<0.05)$. Blood lactate concentration was higher in the climbing group $(p<0.05)$. Other parameters shown in Table 1 were identical in both groups. Intra-erythrocyte lactate concentration was positively correlated with plasma lactate concentration in the climbing group $(r=0.80, p<0.001$; Fig. 1). Erythrocyte MCT1 content was significantly higher in the climbing group than in the sedentary group $(p<0.05$; Fig. 2A). In addition, when MCT1 content was measured separately in young and old erythrocytes, young erythrocytes tended to have more MCT1 than old erythro-

Table 1. Body weights and hematological parameters in sedentary and climbing rats.

\begin{tabular}{lcc} 
& \multicolumn{2}{c}{ Groups } \\
\cline { 2 - 3 } & Sedentary & Climbing \\
\hline Body weight $(\mathrm{g})$ & $374 \pm 2$ & $366 \pm 3^{*}$ \\
$\mathrm{RBC}$ counts $\left(10^{4} \cdot \mu \mathrm{l}^{-1}\right)$ & $766 \pm 18$ & $780 \pm 6$ \\
$\mathrm{Hct}(\%)$ & $44.8 \pm 0.8$ & $45.7 \pm 0.2$ \\
$\mathrm{Hb}\left(\mathrm{g} \cdot \mathrm{dl} l^{-1}\right)$ & $1.00 \pm 0.02$ & $1.02 \pm 0.02$ \\
{$[\mathrm{La}](\mathrm{mm})$} & $1.7 \pm 0.2$ & $2.6 \pm 0.4^{*}$ \\
\hline
\end{tabular}

Values are presented as means $\pm \mathrm{SE}$ for rats in each group $(n=13-15)$. [La] is measured during the climbing period of the dark cycle. Other values were measured at sacrifice. *Significantly different from the sedentary group $(p<0.05)$. RBC, erythrocyte; Hct, hematocrit; Hb, hemoglobin; [La], blood lactate concentration. 


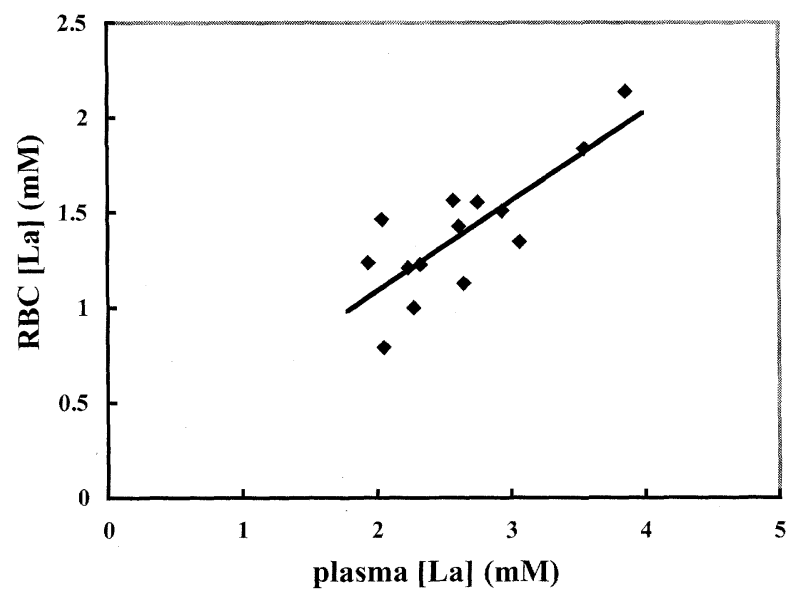

Fig. 1. Relationship between intra-erythrocyte and plasma lactate concentrations in the climbing rats $(n=14)$ during the climbing period. The curve was $y=0.48 x+0.11, r=0.80, p<0.001$. RBC, erythrocytes; [La], lactate concentration.

MCT1 protein contents
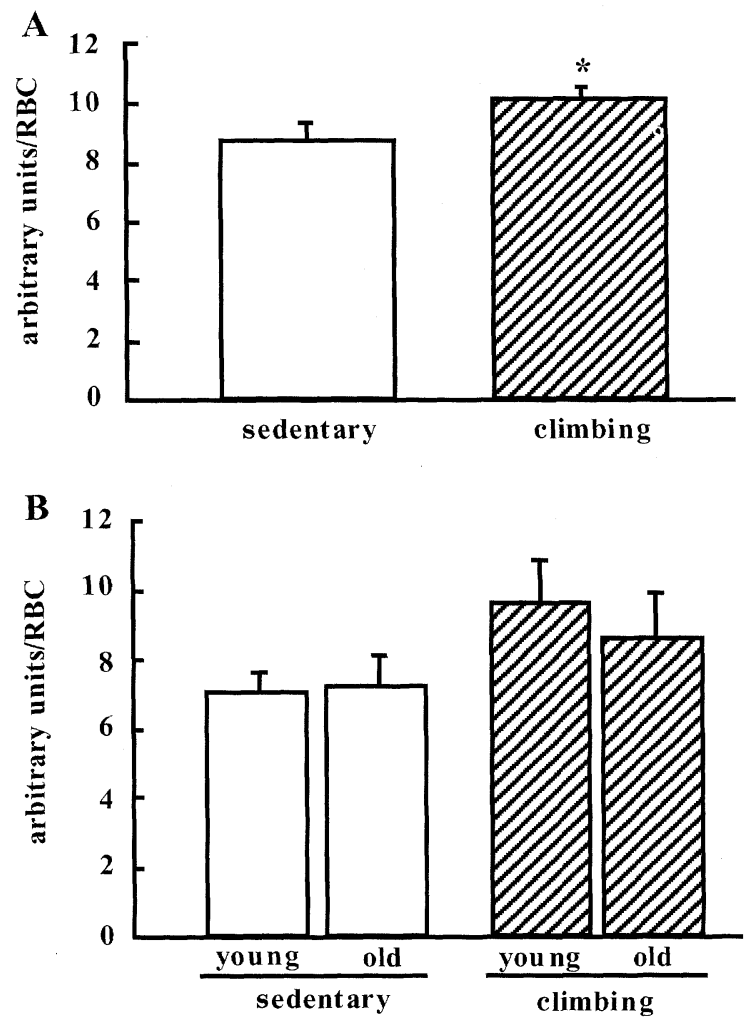

Fig. 2. MCT1 contents in total erythrocytes (A: $n=12)$ and in young $v s$. old erythrocytes (B: $n=7)$ in rats. Values are presented as means \pm SE. *Significantly different from the sedentary group $(p<$ $0.05)$. 
cytes in the climbing group (not significance), but no such tendency was seen in the sedentary group (Fig. 2B).

\section{DISCUSSION}

We demonstrated for the first time that erythrocyte MCT1 content was increased with sustained voluntary climbing exercise for four weeks in rats. Other studies in humans and rats have demonstrated that MCT1 contents in skeletal muscles and the heart are increased by endurance- and high-intensity-training [13-15]. In addition chronic electrical stimulation increased skeletal muscle MCT1 content [16]. During muscle contraction, a large amount of lactate is produced in the muscles, which is distributed to various tissues and cells [1]. MCT1 greatly contributes to bidirectional lactate movement across the cell membranes; thus, lactate transport capacity largely depends on the membrane MCT1 content [13-16]. In the present study, the climbing exercise elevated lactate concentrations in blood and erythrocyte, suggesting that lactate movement across erythrocyte membranes is increased by the climbing exercise. Although the mechanisms are still obscure, MCT1 content in tissues including erythrocytes may be up-regulated with the increase in lactate movement.

Young rather than old erythrocytes had a tendency toward a greater concentration of MCT1 in the climbing group. In addition, the ratio of young erythrocytes to total erythrocytes was slightly higher in the climbing group than in the sedentary group, indicating that the life span of erythrocytes in the climbing group is shorter than that of the sedentary group (data not shown). In other lines of study, there is evidence that younger erythrocytes have more membrane protein sodium-potassium pump [17]. However, climbing exercise did not heighten MCT1 content in younger erythrocytes, which may be because the intense is too low or the training period is too short and so on. Therefore, it is desirable that studies can carry out under some conditions such as marked increase in blood lactate concentration. Then climbing exercise may increase MCT1 in younger erythrocytes more clearly and decrease the half-life of erythrocytes, which in turn increases the ratio of young erythrocytes to total erythrocytes and partly contributes to higher erythrocyte MCT1 content.

By the way erythrocytes are produced by differentiation and self-replication of the stem cells in bone marrow. After released into peripheral circulation, mature erythrocytes lose nucleus and cannot synthesize protein any further. Thus, the synthesis and membrane incorporation of MCT1 protein in erythrocyte are induced during erythropoiesis in the bone marrow. Previous studies described that erythrocyte membrane protein content such as glucose transporter 1 [18] and sodium-potassium pump [17, 19] is regulated by environmental changes in plasma, maybe interstitial glucose or intracellular sodium, and the regulation is occurred during erythropoiesis. Erythrocyte MCT1 would also be already increased at the pre-erythrocyte stage of erythroid cell.

Physiological importance of increase in erythrocytes MCT1 has not been well understood. As mentioned above, MCT1 in skeletal muscles and the heart plays a physiological important role in facilitating lactate movement across the sarcolemmal membrane, subse- 
quently removal of lactate from circulation and its oxidation. Plasma and erythrocytes have important role in carrying lactate from active muscles leading to utilizing tissues. It is advantageous to extrude lactate from the contracting muscles to limit reductions in muscle pH. During exercise ca. $30 \%$ of the blood lactate is taken up by erythrocytes [4] which provide an additional space for translocation of lactate and hydrogen ions from plasma. Rapid uptake of these ions by the erythrocytes would aid in establishing a gradient between plasma and interstitial fluid [20,21]. As MCT1 bears $90 \%$ of erythrocyte lactate transport [4], erythrocyte MCT1 plays a central role of the physiological roles in lactate delivery. Therefore, in the present study, an increase in MCT1 content in erythrocyte by climbing exercise may contribute to a larger amount of lactate uptake and rapidly carrying and removal of lactate from the circulation. Concerning the physiological role of erythrocyte MCT1 increment, we will need to study further.

In summary, erythrocyte MCT1 content was increased with the sustained climbing exercise, which might be due to the increase in blood lactate concentration. However, maybe because the exercise was relatively mild, the elevation of blood lactate was not remarkable, which might limit the increase in erythrocyte MCT1. Although further studies are required to clarify the details, the findings suggest that habitual voluntary climbing exercise affects regulation of MCT1 content in erythrocytes.

\section{REFERENCES}

1. Brooks, G.A. (2000): Intra- and extra-cellular lactate shuttle. Med. Sci. Sports Exerc., 32, 790-799.

2. Smith, E.W., Skeleton, M.S., Kremer, D.E., Pascoe, D.D., and Gladden, L.B. (1997): Lactate distribution in the blood during progressive exercise. Med. Sci. Sports Exerc., 29, 654-660.

3. Smith, E.W., Skeleton, M.S., Kremer, D.E., Pascoe, D.D., and Gladden, L.B. (1998): Lactate distribution in the blood during steady-state exercise. Med. Sci. Sports Exerc., 30, 1424-1429.

4. Skelton, M.S., Kremer, D.E., Smith, E.W., and Gladden, L.B. (1995): Lactate influx into red blood cells in athletic and nonathletic species. Am. J. Physiol., 268, R1121-R1128.

5. Deuticke, B. (1989): Monocarboxylate transport in red blood cell: Kinetics and chemical modification. Methods Enzymol., 173, 300-329.

6. Juel, C., and Helestrap, A.P. (1999): Lactate transport in skeletal muscle-role and regulation of the monocarboxylate transporter. J. Physiol., 517, 633-642.

7. Notomi, T., Okimoto, N., Okazaki, Y., Tanaka, Y., Nakamura, T., and Suzuki, M. (2001): Effects of tower climbing exercise on bone mass, strength, and turnover in growing rats. J. Bone Miner. Res., 16, 166-174.

8. Matsuo, T., Mizushima, Y., Fujie, M., and Suzuki, M. (1999): Effect of voluntary resistance exercise on mitochondrial heme biosynthesis in rat liver. J. Clin. Biochem. Nutr., 27, 151-159.

9. Buono, M.J., and Yeager, J.E. (1986): Intraerythrocyte and plasma lactate concentration during exercise in humans. Eur. J. Appl. Physiol., 55, 326-329.

10. Pool, R.C., and Halestrap, A.P. (1988): Reconstitution of the L-lactate carrier from rat and rabbit erythrocyte plasma membranes. Biochem. J., 254, 385-390.

11. Carpenter, L., Poole, R.C., and Halestrap, A.P. (1996): Cloning and sequencing of the monocarboxylate transporter from mouse Ehrlich Lettre tumor cell confirms its identity as MCT1 and demonstrates that glycosylation is not required for MCT1 function. Biochim. Biophys. Acta, 1279, 157163.

12. Kim, Y.B., Nakajima, R., Matsuo, T., Inoue, T., Sekine, T., Komuro, M., Tamura, T., Tokuyama, K., and Suzuki, M. (1996): Gene expression of insulin signal-transduction pathway intermediates is lower in rats fed a beef tallow diet than in rats fed a safflower oil diet. Metabolism, 45, 1080-1088.

13. Bonen, A., McCullagh, K.J.A., Putman, C.T., Hultman, E., Jones, N.L., and Heingenhauser, G.J.F. 
(1998): Short-term training increases human muscle MCT1 and femoral venous lactate in relation to muscle lactate. Am. J. Physiol., 274, E102-E107.

14. Baker, S.K., McCullagh, K.J., and Bonen, A. (1998): Training intensity-dependent and tissue-specific increases in lactate uptake and MCT-1 in heart and muscle. J. Appl. Physiol., 84, 987-994.

15. Pilegaard, H., Domino, K., Noland, T., Juel, C., Hellsten, Y., Halestrap, A.P., and Bangsbo, J. (1999): Effect of high-intensity exercise training on lactate $/ \mathrm{H}^{+}$transport capacity in human skeletal muscle. Am. J. Physiol., 276, E255-E261.

16. McCullagh, K.J.A., Poole, R.C., Halestrap, A.P., Tipton, K.F., O'Brien, M., and Bonen, A. (1997): Chronic electrical stimulation increases MCT1 and lactate uptake in red and white skeletal muscle. Am. J. Physiol., 273, E239-E246.

17. Kaji, D.M., Thakkar, U., and Kahn, T. (1981): Glucocorticoid-induced alterations in the sodium potassium pump of the human erythrocyte. J. Clin. Invest., 68, 422-430.

18. Hark, S.I., Behmand, R.A., and Arafah, B.M. (1991): Chronic hyperglycemia increases the density of glucose transporters in human erythrocyte membranes. J. Clin. Endocrinol. Metab., 72, 814-818.

19. Cheng, J.T., Kahn, T., and Kaji, D.M. (1984): Mechanism of alteration of sodium potassium pump of erythrocytes from patients with chronic renal failure. J. Clin. Invest., 74, 1811-1820.

20. Deuticke, B. (1982): Monocarboxylate transport in erythrocytes. J. Membr. Biol., 70, 89-103.

21. Roth, D.A. (1991): The sarcolemmal lactate transport: Transmembrane determinants of lactate flux. Med. Sci. Sports Exerc., 23, 925-934. 